\title{
Improving Cancer Outcomes through Electrochemical Biosensing of Early Diagnosis/Prognosis Biomarkers in Human Biopsies ${ }^{\dagger}$
}

\author{
José Manuel Pingarrón 1,*, Susana Campuzano ${ }^{1}$, Rebeca Magnolia Torrente-Rodríguez ${ }^{1}$, \\ Víctor Ruiz-Valdepeñas Montiel ${ }^{1}$, Eva Vargas ${ }^{1}$ and Rodrigo Barderas ${ }^{2}$ \\ 1 Departamento de Química Analítica, Facultad de CC. Químicas, Universidad Complutense de Madrid, \\ 28040 Madrid, Spain; susanacr@quim.ucm.es (S.C.); rebeca.magnolia@gmail.com (R.M.T.-R.); \\ victor_lega90@hotmail.com (V.R.-V.M.); evargas_orgaz@hotmail.com (E.V.) \\ 2 Departamento de Bioquímica y Biología Molecular I, Facultad de CC. Químicas, Universidad Complutense \\ de Madrid, 28040 Madrid, Spain; rbarderas@quim.ucm.es \\ * Correspondence: pingarro@quim.ucm.es \\ + Presented at the 5th International Symposium on Sensor Science (I3S 2017), Barcelona, Spain, \\ 27-29 September 2017.
}

Published: 29 November 2017

Cancer is the second leading cause of death after cardiovascular diseases and responsible for over 8 million deaths worldwide. Although substantial progress has been made in the prevention and treatment of different cancer types, its incidence and prevalence is increasing in recent years. Therefore, the development of efficient, simple, quantitative and disposable devices with short response times, low cost, and that are suitable to perform decentralized and reliable determination of early diagnosis cancer biomarkers could help to reduce cancer mortality due to its detection at early stages, when the disease can be efficiently treated and cured in more than $90 \%$ of cancer patients.

Within this context, the main characteristics of novel biosensing scaffolds for the determination of cancer related-miRNAs [1-3] and autoantibodies [4] against tumor associated antigens (TAAs), relevant biomarkers considered for both early diagnosis and prognosis, will be presented. The developed methodologies, based on the coupling of attractive bioreceptors and bioassay formats, functionalized magnetic microcarriers, and electrochemical detection at disposable transducers, have demonstrated practical applicability for the accurate determination of the endogenous concentration of the target analytes in solid (fresh and FFPE breast human tissues) and liquid (human serum from colorectal and ovarian cancer patients) biopsies.

The developed easy handling single- or multiplexed platforms, readily applied to the determination of other biomarkers, provided results in agreement with conventional methodologies but with lower cost and in remarkably shorter times. These interesting features make them suitable alternatives in the implementation of user-friendly and affordable devices, particularly attractive to perform routine determinations in both clinical and basic research settings to improve cancer diagnosis and prognosis.

\section{References}

1. Torrente-Rodríguez, R.M.; Ruiz-Valdepeñas Montiel, V.; Campuzano, S.; Farchado-Dinia, M.; Barderas, R.; San Segundo Acosta, P.; Montoya, J.J.; Pingarrón, J.M. Fast electrochemical miRNAs determination in cancer cells and tumor tissues with antibody-functionalized magnetic microcarriers. ACS Sensors 2016, 1, 896-903.

2. Torrente-Rodríguez; Campuzano, S.; Ruiz-Valdepeñas Montiel, V.; Montoya, J.J.; Pingarrón, J.M. Sensitive electrochemical determination of miRNAs based on a sándwich assay onto magnetic microcarriers and hybridization chain reaction amplification. Biosens. Bioelectron. 2016, 86, 516-521. 
3. Torrente-Rodríguez; Campuzano, S.; Ruiz-Valdepeñas Montiel, V.; Sagrera, A.; Domínguez-Cañete, J.J.; Vargas, E.; Montoya, J.J.; Granados, R.; Sánchez-Puelles, J.M.; Pingarrón, J.M. Electrochemical miRNAs determination in formalin-fixed, paraffin-embedded breast tumor tissues association with HER2 expression. J. Biotecnol. Biomed. Eng. 2016, 3, 1064.

4. Garranzo-Asensio, M.; Guzmán-Aranguez, A.; Povés, C.; Fernández-Aceñero, M.J.; Torrente-Rodríguez, R.M.; Ruiz-Valdepeñas Montiel, V.; Domínguez, G.; San Frutos, L.; Rodríguez, N.; Villalba, M.; et al. Toward liquid biopsy: determination of the humoral immune response in cancer patients using HaloTag fusion protein-modified electrochemical bioplatforms. Anal. Chem. 2016, 88, 12339-12345.

(C) 2017 by the authors. Licensee MDPI, Basel, Switzerland. This article is an open access article distributed under the terms and conditions of the Creative Commons Attribution (CC BY) license (http://creativecommons.org/licenses/by/4.0/) 